\title{
A survey of online social networking used to support health awareness campaigns in the City of Johannesburg metropolitan municipality
}

\author{
Authors: \\ Karin Eloff ${ }^{1}$ \\ Cornelius J. Niemand ${ }^{1}$ \\ Affiliations: \\ ${ }^{1}$ Centre for Information and \\ Knowledge Management, \\ University of Johannesburg, \\ South Africa

\section{Correspondence to:} \\ Cornelius Niemand \\ Email: \\ corn@uj.ac.za \\ Postal address: \\ PO Box 524, Auckland Park \\ 2006, South Africa \\ Dates: \\ Received: 22 Jan. 2013 \\ Accepted: 03 May 2014 \\ Published: 21 Aug. 2014 \\ How to cite this article: \\ Eloff, K. \& Niemand, \\ C.J., 2014, 'A survey of \\ online social networking \\ used to support health \\ awareness campaigns in \\ the City of Johannesburg \\ metropolitan municipality', \\ SA Journal of Information \\ Management 16(1), Art. \\ \#550, 6 pages. http://dx.doi. \\ org/10.4102/sajim.v16i1.550

\section{Copyright: \\ C 2014. The Authors. Licensee: AOSIS \\ OpenJournals. This work is licensed under the Creative Commons Attribution License.}

Read online:

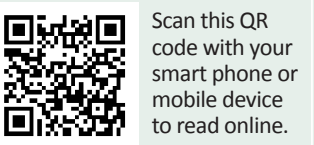

Background: The Department of Health $(\mathrm{DoH})$ at the City of Johannesburg metropolitan municipality in South Africa develops various health awareness campaigns aimed at creating awareness of general health risks within the Johannesburg area. According to staff members of the DoH, the resources utilised in the current campaigns fail to reach a sufficiently broad audience and the campaigns struggle to deliver the intended messages. Furthermore, the development and implementation of campaigns are time consuming and costly.

Objectives: This research focused on how online social networking (OSN) can support health awareness campaigns for the $\mathrm{DoH}$ in the Johannesburg region. OSN may be regarded as a tool that will assist the $\mathrm{DoH}$ to reach a wider audience, send health-related messages and provide a two-way communication channel.

Method: The research used an exploratory research design with a purposive non-probability sample. A survey was used as the data collection instrument. Statistical analysis was performed on the data obtained from the surveys.

Results: The results indicate that the DoH can benefit from the use of OSN in health promotion campaigns. The benefits include, but are not limited to, an increase in engagement with the target market, ease of use and reach within the specified audience.

Conclusion: Although there are numerous advantages associated with the integration of OSN by the $\mathrm{DoH}$, the $\mathrm{DoH}$ needs to develop training and development programmes for OSN to encourage its use by DoH staff members. The main aim of the programmes is to create internal OSN capabilities to support the OSN strategy.

\section{Introduction}

According to Smith (2011), online social networking (OSN) is 'one of the greatest innovations in the Internet today. The Internet provides the means for information dissemination, selling products and playing games'.

Glenn (2013) postulates that the use of OSN by Internet users has greatly increased over the last decade. The most popular, according to the Glenn (2013), may include, but are not limited to, Facebook, Twitter, MySpace and LinkedIn. According to various studies including Smith (2011) and Tau (2011:10) users of OSN identify them as a tool to reach wide audiences, broadcast a message, communicate with relatives and build relationships and connect with people.

A number of not-for-profit organisations and government agencies worldwide have started to implement OSN to share information and knowledge and communicate with their target market utilising the digital channels created by OSN. The Centre of Disease Control (CDC) is an example of a health-related department in the United States of America that makes use of OSN to promote awareness about current health promotion campaigns. The CDC recognises both advantages, including an increase in engagement with the target market, ease of use and reach, and disadvantages, including costs and time constraints with regard to use of OSN. The CDC has developed a toolkit to promote the use of online social networking. This toolkit provides guidance regarding OSN and lessons on how participation in and incorporation of OSN into government agencies to promote health promotion campaigns, can be achieved (Brodalski et al. 2011).

The Department of Health $(\mathrm{DoH})$ at the City of Johannesburg metropolitan municipality in South Africa is continuously developing various kinds of health promotion campaigns that differ depending on the health requirement in the particular region. The present techniques that the DoH utilises include face-to-face interaction, information sessions, education and communication materials such as pamphlets and posters and other vocal and print media that can include 
press releases and radio broadcasts. The DoH made use of OSN sites in previous non-health-related campaigns such as the FIFA World Cup ${ }^{\mathrm{TM}}$ in 2010. These OSN sites were utilised to communicate and share information and knowledge regarding the events taking place during the World Cup. After the implementation of OSN throughout the World Cup, the DoH considered making use of OSN sites more extensively for health awareness promotion (Tau 2011:105-106).

Taking the aforesaid into consideration, the main objective of this study was to investigate:

- The extent to which OSN supports information sharing within health awareness promotion campaigns by the DoH.

- The types of social media channels that are currently being utilised by the DoH.

- Possible OSN sites or channels that could be implemented by the DoH.

The literature reviewed (with specific emphasis on the reports generated by the CDC in the United States of America) suggests that the DoH can achieve a variety of benefits by incorporating OSN into its communication strategy; the benefits include an increase in engagement with the target market, ease of use and reach from the implementation of OSN to promote current health promotion campaigns. However, within the DoH context, campaign managers lack practical experience in the use of OSN tools and are therefore not able to recognise the benefits that OSN holds for current health promotion campaigns.

\section{Literature review}

\section{The development of the Internet and Web}

To grasp the full extent of the use and integration of online social networking (OSN) within the new digital economy a brief overview of the development of the Internet and the Web is needed.

An earlier 'birthday' for the Internet may hark back to 1972, when the first connection was opened between the Advanced Research Project Agency Network (ARPANET) and another network, ALOHAnet in Hawaii, or even to 1969, when the first four nodes of ARPANET were connected to each other, or to the meeting of the Association of Computing Machinery in 1967, when Larry Roberts read the first public paper describing the ARPANET design. It is possible to go back even further, though, to Paul Baran's work on robust networks for research and development in the mid-1960s, or (albeit less convincingly) to the launch of Sputnik in 1957, which led to the convening of the Advanced Research Projects Agency (Thomas 1999:682).

Taking the aforesaid into consideration and according to Mowery (2002), however, the history of the Internet's development should ideally be divided into three phases (see Table 1).
TABLE 1: The history of the Internet.

\begin{tabular}{ll}
\hline Time period & Critical developments \\
\hline $1960-1985$ & $\begin{array}{l}\text { Invention of digital packet-switching and associated } \\
\text { standards and protocols } \\
\text { Birth of Internet self-governance institutions }\end{array}$ \\
$\begin{array}{l}\text { Growth of NSFNET and parallel private infrastructure } \\
\text { Growth in installed base of PCs and local area networks }\end{array}$ \\
$\begin{array}{l}\text { 1996- the present } \\
\begin{array}{l}\text { Diffusion of the World Wide Web } \\
\text { Privatisation of the Internet infrastructure and } \\
\text { commercialisation of the Internet content }\end{array}\end{array}$ \\
\hline
\end{tabular}

NSFNET, The National Science Foundation Network; PCs, personal computers

Thus, it can be said that the Internet is a large network of networks; in other words, the Internet is a maze of phones and cable lines, satellites and network cables that interconnects computers around the world, thereby creating a global network through which any computer can communicate with any other computer, as long as they are both connected to the Internet.

Many people use the terms 'Internet' and 'Web' interchangeably, whilst they are, in fact, not even synonymous. The Internet and the Web are two separate, yet related, entities. The Word Wide Web, commonly referred to as 'the Web', can be seen as the multimedia section of the Internet, as it allows the individual surfer to explore a seemingly unlimited worldwide digital 'Web' of information, expanding by tens of thousands of websites each day. The Web presents the user with a way in which to represent information on the Internet. Information resources are located by using a uniform resource locator. This is an informationsharing model that is built on top of the Internet. The Web, therefore, needs the Internet, and not vice versa. The Web uses the hypertext transfer protocol to share information across the Internet. The Web also uses browsers, such as Internet Explorer, to access Web documents called 'Web pages', which are linked via hyperlinks. Web documents can also contain graphics, sound, text and video (Webopedia 2002).

\section{Online social networking}

According to Taprial and Kanwar (2012), OSN refers to 'the media that allows one to be social, or get social online by sharing content, news, photos etc. with other people'.

The Internet has directly influenced the way that people perform their daily activities over the past decade. Out of all online users, $90 \%$ are actively involved in some type of OSN site (Taprial \& Kanwar 2012). OSN is not only a tool that is utilised by individuals for personal use but also by both profit and not-for-profit organisations for marketing purposes and as a business tool (for recruitment, communication and brand-building exercises). According to Lusted (2011), there are numerous advantages and challenges when making use of OSN sites. Advantages that organisations can gain from the implementation of OSN in general include increased communication and collaboration, improved education in terms of the topic at hand and building relationships. Furthermore, OSN assists with the broadcasting of messages over a large geographical area and allows participation to occur. There are also potential challenges involved in the implementation of OSN. These challenges include privacy issues, lack of understanding of the OSN tools and features and having a negative impact on staff productivity. 
Furthermore, staff in general are generally resistant to change and will therefore not immediately be on board with adjustments to their routine activities. OSN sites can also be very time consuming for staff to update and maintain and to respond to messages sent via OSN sites (Lusted 2011). Bearing in mind the management of potential challenges, OSN has several advantages for health promotion campaigns within the DoH.

\section{Statement of the problem}

This study investigated the potential usage of OSN by the $\mathrm{DoH}$ as part of health promotion campaigns. OSN is a cost-effective tool that can reach a large target audience in a short period of time. The tools used by the DoH to share knowledge regarding campaigns were identified and the most unsuccessful tools were assessed. In addition, the research identified reasons why the DoH is currently not making use of OSN to promote current health promotion campaigns.

\section{Research design Sample}

This study utilised a purposive sample. The objective of the study was to determine the use of OSN in the promotion of health campaigns by the DoH. The gathering of data was done by firstly identifying the respondents' knowledge regarding the definition of the concept, secondly recognising the respondents' awareness in terms of the benefits of OSN and finally determining their active involvement in OSN in both a personal and a professional environment. In order for the purposive sample to be successful, the respondents selected were managers of health promotion campaigns run by the $\mathrm{DoH}$ for the reason that they had direct involvement in the development of health promotion campaigns.

The DoH consists of two main sub-divisions, namely the central health department and the regional health department. It is the responsibility of the central health department to develop and implement health promotion campaigns. The central health department has five sub-units, namely management support, primary health care, public health, HIV / AIDS and environmental health units.

A total of 30 questionnaires were sent to the respondents. Of the 30 questionnaires, 18 legible and complete questionnaires were returned, which resulted in a $60 \%$ response rate. Of the 18 completed questionnaires one questionnaire was completed by each of these sub-units together with three questionnaires by the regional health department. The campaign managers of the following campaigns also completed a questionnaire: HIV/AIDS, TB, malaria, teenage pregnancy, suicide prevention and early childhood education centres.

\section{Data collection instrument}

The questionnaire utilised as the data collection instrument was developed with the main intention of evaluating the extent to which managers utilise OSN in the promotion of health campaigns by the DoH. The questionnaire included both closed-ended questions and multi-option questions. The questionnaire firstly focused on how the respondents fit into the DoH in terms of education and experience gained in the specific field of health promotion campaigns. In addition, it also focused on gathering general information regarding the tools utilised in health promotion campaigns and how successful these tools were in reaching the desired objectives.

The questionnaire then focused on each respondent's knowledge regarding OSN as well as the amount of time the respondents spend on OSN. A further goal of the questionnaire was to ascertain the benefits that OSN can hold for health promotion campaigns.

\section{Data analysis and results}

Statistical analysis was performed on the data obtained from the questionnaire. This identified the utilisation of OSN inside health promotion campaigns and the number of respondents actively involved in OSN, together with the average time spent on OSN in both a personal and a professional capacity. Furthermore statistical analysis, utilising statistical analysis tools, assisted with the identification of the respondents' opinions in terms of the possible effectiveness of OSN in health promotion campaigns and the percentage of time spent inside the DoH by respondents when working on health promotion campaigns.

\section{Results}

Figure 1 indicates that $56 \%$ of the respondents have a postgraduate qualification in a health-related field. The results indicated that $14 \%$ of the respondents have worked in a health-related institution for more than six years.

The DoH currently makes use of pamphlets, posters, print media releases including newspapers and magazines and vocal media releases including radio stations, TV, door-todoor campaigns, banners, health talks, fun runs and talk clinics to share knowledge of the health promotion campaigns. Figure 2 depicts that $78 \%$ of the respondents agreed that the different media mechanisms were successfully implemented, whereas $11 \%$ believed that that was not the case.

According to the respondents, pamphlets and media releases were the two most unsuccessful media that are currently implemented to promote health promotion campaigns.

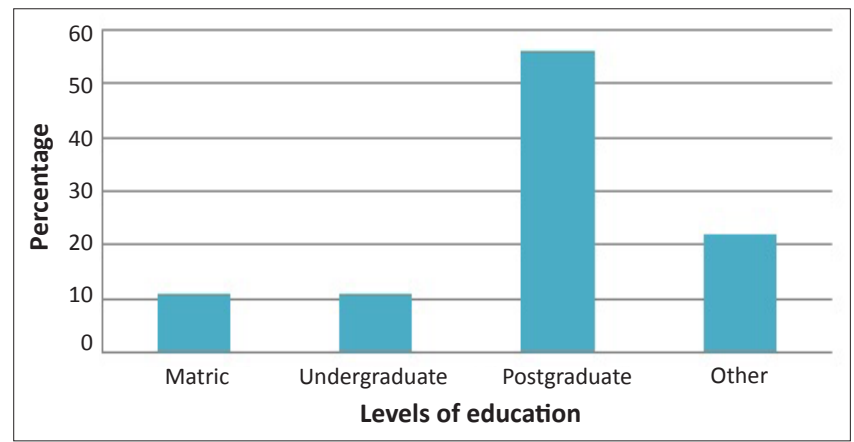

FIGURE 1: Educational levels. 
Figure 3 provides a clear illustration of the reasons for current tools being unsuccessful. The majority of the respondents $(56 \%)$ selected a lack in physical storage space as the main reason for the current tools being unsuccessful; 33\% of the respondents believed that current tools are ineffective in reaching the target audience, $22 \%$ stated that current tools are not cost effective, $33 \%$ of the respondents believed that current tools are not able to share the message clearly and $38 \%$ believed a lack in interaction made them unsuccessful.

All participants indicated that they did know what OSN was. Respondents had to select from a list which OSN tools they recognised. None of the respondents selected all the OSN tools. A large portion of the respondents indicated Facebook, Twitter and MySpace as OSN tools. Respondents disregarded the following as OSN tools: blogs, wikis, LinkedIn and discussion groups.

Figure 4 displays reasons that OSN can possibly be successful in health promotion campaigns. The majority of

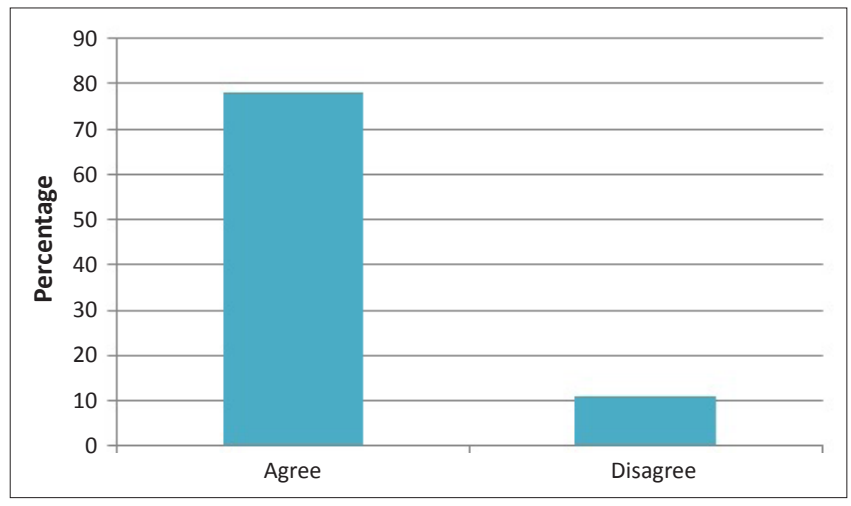

FIGURE 2: Current media successfully implemented.

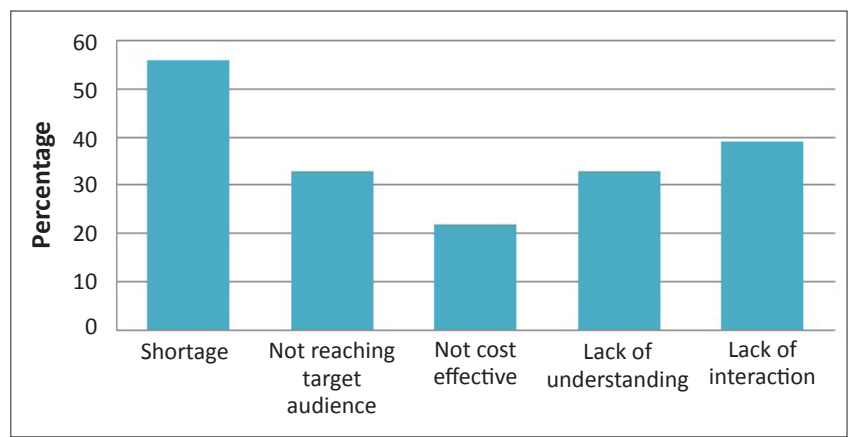

FIGURE 3: Reasons for current media being unsuccessful.

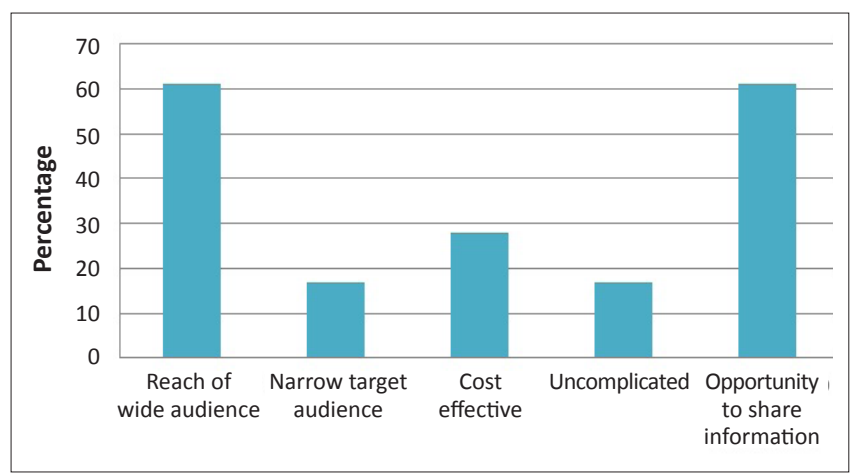

FIGURE 4: Reasons online social networking can possibly be successful in health promotion campaigns. the respondents $(61 \%)$ stated that reaching a wider audience is the key reason for success, $61 \%$ also believed that OSN can allow health promotion campaigns to share information more easily, $28 \%$ believed that OSN is a cost-effective option, $17 \%$ selected uncomplicated as an option and the other $17 \%$ believed that OSN will allow health promotion campaigns to focus on a narrower target market.

\section{Discussion of the findings}

The results indicate that the majority of respondents are highly educated in their specific health field. The majority of the respondents are not only educated but have also completed six or more years in a health-related field and therefore have gained a sufficient amount of experience.

Each individual health promotion campaign has its own target market that the campaign managers focus on. Each campaign focuses on both men and women. It is therefore important to select the most appropriate tool to promote the health promotion campaigns for both men and women. The DoH presently makes use of pamphlets, posters, print media releases and vocal media releases to promote current health promotion campaigns. Some of these media, for example door-to-door campaigns, are more effective in comparison with other media, such as pamphlets. It is important to evaluate which of these media are the most unsuccessful and could be replaced with an OSN tool. Pamphlets and media releases have been identified by the respondents as being the most unsuccessful. Some of the possible reasons include a lack of physical storage space, ineffective reach within the target audience, high expenses, lack of understanding by the community for the reason of it only being a one-directional communication channel, lack of interaction and other reasons such as citizens being illiterate or not understanding the language in which the pamphlets and media releases are presented.

Pamphlets and media releases can be eliminated and the DoH could rather implement OSN tools to promote current health campaigns. OSN sites provide a variety of features for users. Some OSN sites such as Facebook allow for a two-way communication channel through which users can interact with one another. Other OSN sites such as YouTube provide the opportunity for users to share a message with videos in order to assist users who are not computer literate, allowing the intended message to be more easily understood.

The results indicated that the respondents were aware of the concept of OSN but it is clear that these respondents are not fully aware of the different OSN tools. This may be the major reason why the respondents have not implemented OSN to share knowledge regarding health promotion campaigns. There is a lack in understanding of what online social media are and the online social media tools that could be utilised to share knowledge. It is essential for the health promotion campaign managers to be actively involved with different OSN tools to identify and recognise the benefits they can hold for a health promotion campaign as well as to recognise the challenges of OSN. 
Online social networking does not need any physical storage space because it occurs via the Internet; it is utilised today by millions of users worldwide. OSN is also inexpensive but requires suitable staff to make updates. OSN makes a two-way communication process possible and if any misunderstandings do occur questions may be received and answered; it is also possible to conduct the communication process in more than one language.

Together with all the advantages, like it being an inexpensive communication channel, there are also some restrictions that can limit the DoH in implementing OSN tools. Some of these limitations can include a lack of Internet access, computer illiteracy and a lack of education.

\section{Recommendations}

An online social media strategy (OSMS) is suggested for the $\mathrm{DoH}$, allowing OSN sites to be implemented and managed effectively and efficiently. The OSMS consists of the following four steps: planning, managing, implementing and feedback.

In the planning stage management will need to identify the problem at hand. The problem, for example, can be that current traditional media being utilised to promote health promotion campaigns are ineffective.

The second stage of the OSMS is the management of the problem. This stage focuses on the development of a few solutions. It is essential to list all the possible solutions together with the possible risks and the probability that these risks might occur in the near future. Identifying the risks together with their probabilities will assist the DoH to select the most appropriate solutions. There are a variety of solutions that the DoH can implement in order to eliminate media releases and posters as a current tool and replace these with OSN. In order for the DoH to successfully implement OSN to promote current health promotion campaigns, a few actions are recommended. These actions can include the development of training and development programmes to improve involvement from higher management and create awareness of the various OSN tools. The training and development programmes can educate the staff inside the DoH regarding the OSN tools and how these tools can be incorporated into health promotion campaigns in order to increase the success rate. Staff should be encouraged, after undergoing intensive training and education in the OSN tools, to test and implement the most appropriate tool. Creating awareness of the benefits of OSN tools will allow campaign managers to be encouraged and to experiment with these tools in different scenarios within different target markets.

The third step of the OSMS is based on the implementation of the selected solution, that is a specific OSN channel for a specific target market. Once the solution has been selected, a pilot event should be created. The pilot event will identify any potential problem areas associated with the selected channel of communication utilised for the specific target market. Once all problem areas have been identified and rectified, the utilisation of the solution may be replicated to other campaigns.

Feedback is the final stage and focuses mainly on the success of the OSN site. The feedback can be received from both staff inside the organisation and citizens making use of OSN to receive information regarding health promotion campaigns. Staff can provide feedback on a variety of aspects, including the physical usage of the OSN site (via various analytical tools like Google Analytics), the target audience that has been reached and the awareness that has been created. The OSMS, if implemented correctly and successfully, can assist the DoH to share information effectively in health promotion campaigns.

\section{Limitations of the study}

This study is limited to only managers of certain health promotion campaigns inside the $\mathrm{DoH}$ and not all the campaigns that are currently in place. The sample of the respondents meant that the study focused on top and middle managers' knowledge and experience gained in terms of OSN and information sharing.

\section{Conclusion}

The DoH is currently still utilising traditional media to share knowledge about health promotion campaigns. Some of these traditional media, with specific reference to pamphlets, are less effective than others, such as door-to-door campaigns. There are a variety of OSN sites available that can assist the DoH to share information about its health promotion campaigns.

The results of the study suggest that respondents lack practical experience in the use of OSN and therefore are incapable of identifying the benefits of OSN for health promotion campaigns.

It would be beneficial for the DoH to develop an OSN training and development programme. This programme will assist the staff in learning more about OSN tools and features together with the ability to select the most appropriate OSN sites for each specific health promotion campaign. This training and development programme should also motivate and highlight the importance of the use of OSN. An online social media strategy is recommended as it will allow the $\mathrm{DoH}$ to implement OSN more efficiently and effectively in four basic steps.

A suggestion for further research is to identify the specific OSN sites that are to be utilised to reach specific target markets. Health promotion managers have to know what the needs for each individual location are and how knowledge 
can be effectively shared with a specific target market. It is therefore essential to be able to identify which tools for health promotion campaigns are the most appropriate in specific locations.

\section{Acknowledgements \\ Competing interests}

The authors declare that they have no financial or personal relationship(s) that may have inappropriately influenced them in writing this article.

\section{Authors' contributions}

C.J.N. (University of Johannesburg) was the study leader and K.E. (University of Johannesburg), the student. Both authors made equal contributions in terms of the writing and analysis of this article.

\section{References}

Brodalski, D., Brink, H., Curtis, J., Diaz, S., Schindelar, J., Shannon, C. et al., 2011, 'The Health Communicators social media toolkit', in The health communicator's socia media toolkit, viewed 10 December 2012, from http://www.cdc.gov/socialmedia/ tools/guidelines/pdf/socialmediatoolkit_bm.pdf

Glenn, D., 2013, 'Social media use has more than tripled among internet users 65 and older, study finds', SocialTimes, viewed 03 October 2013, from http://socialtimes. com/72-percent-of-online-adults-on-social-networks-pew-study_b133578

Lusted, M.A., 2011, Social networking: MySpace, Facebook and Twitter, ABDO Publishing Company, North Mankato.

Mowery, D.C., 2002, 'Is the Internet a US invention? An economic and technological history of computer networking', in druid, viewed 11 November 2013, from http://www.druid.dk/conferences/nw/paper1/movery.pdf

Smith, B., 2011, 'The great importance of social networking', in Sooper Articles, viewed 12 November 2012, from http://www.sooperarticles.com/internetarticles/spam-articles/great-importance-social-networking-300688.html

Taprial, V. \& Kanwar, P., 2012, Understanding social media, Ventus Publishing, London.

Tau, M.P., 2011, Joburg 2040: Growth and development strategy, City of Johannesburg, Johannesburg.

Thomas, G., 1999, 'Shaping cyberspace - interpreting and transforming the internet', Research Policy 28, 681-698.

Webopdia, 2002, The difference between the Internet and the world wide web viewed 11 November 2013, from http://www.webopedia.com/DidYouKnow/ Internet/2002/Web_vs_Internet.asp 